\title{
(Sub)mm Interferometry Applications in Star Formation Research
}

\author{
Henrik Beuther ${ }^{1}$ \\ Max-Planck-Institute for Astronomy, Königstuhl 17, 69117 Heidelberg, Germany \\ beuther@mpia.de
}

\section{Introduction}

Interferometry at (sub)mm wavelengths is one of the most important tools to study the physical and chemical properties of the youngest and most embedded stages of star formation. High spatial resolution is needed to resolve small-scale structure, e.g., details of accretion disks, molecular outflow or the planet formation processes in low-mass star formation. Furthermore, in high-mass star formation research high spatial resolution is similarly important because the regions are on average more distant, and massive star formation proceeds always in a clustered mode. The (sub)mm wavelengths bands are important because young and deeply embedded stages of star formation are characterized by cold gas and dust temperatures of the order $10 \mathrm{~K}$. At such low temperatures, the peak of the Planck-blackbody curve is at $\lambda_{\max }[\mathrm{mm}]=2.9 / \mathrm{T}=290 \mu \mathrm{m}$ (Wien's law) and one can easily observe the whole Rayleigh-Jeans tail at (sub)mm wavelengths. Therefore, the combined requirement of high spatial resolution and (sub)mm wavelengths focus the research interest to (sub)mm interferometry.

While early exploratory interferometry at even longer wavelengths started already shortly after World War II, real imaging interferometry rather began at cm wavelengths in the 1970s with instruments like the the Very Large Array (VLA) in the USA and the Westerbork Synthesis Array in the Netherlands (in the 1960s). During the 1980s, technical progress allowed to go to even shorter wavelengths, and $\mathrm{mm}$ facilities like the Plateau de Bure Interferometer (PdBI) in Europe, the Berkeley-Illinois-Maryland-Array (BIMA) and the Owens Valley Radio Observatory (OVRO) in the USA, and the Nobeyama Millimeter Array (NMA) in Japan were built to explore the mm wavelength range at high angular resolution. Only a few years ago, in 2003, the Submillimeter Array (SMA) managed to reach the submm window with interferometric techniques, and the Atacama Large Millimeter Array (ALMA) will cover all earth-accessible (sub)mm wavelengths windows from the next decade onwards. 
Each (sub)mm and $\mathrm{cm}$ wavelengths window has its own advantages and disadvantages, and one has to select the right regime according to the scientific questions. For example, at $\mathrm{cm}$ wavelengths, the free-free emission is strong, one finds important maser transitions (e.g., $\mathrm{H}_{2} \mathrm{O}, \mathrm{CH}_{3} \mathrm{OH}$, OH, etc.), a few interesting molecules have easy accessible spectral lines there (e.g., $\mathrm{OH}, \mathrm{NH}_{3}$ ), and one can observe the $21 \mathrm{~cm}$ line of neutral hydrogen there. Going to the $\mathrm{mm}$ regime, the dust continuum emission rises strongly, and one can observe the dense dust and gas cores. Furthermore, the mm regime harbors the low-energy lines of one of the most important astrophysical molecules Carbon Monoxide (CO $J=1-0,2-1)$. In addition to $\mathrm{CO}$, many more complex molecules have important transitions in the $\mathrm{mm}$ regime, and one can trace various physical and chemical properties with the large range of molecular line transitions. Stepping to the submm window, the dust and line emission rises even more strongly $\left(S_{\text {cont }} \propto \nu^{\alpha}\right.$ with $2<\alpha<4, S_{\text {line }} \propto \nu^{5}$ ). Regarding the temperature and density regimes many molecules are sensitive for, the $\mathrm{mm}$ regime rather favors spectral lines emitted from the colder gas $(10<T<50 \mathrm{~K})$, whereas one finds in the submm regime mainly lines from warmer and denser gas $(T>50 \mathrm{~K})$. However, this differentiation is only on average valid, since one finds extremely highly excited lines already at $\mathrm{cm}$ wavelengths (e.g., high $(J, K) \mathrm{NH}_{3}$ inversion lines), and other low-level lines can be observed in the submm regime (e.g., some $\mathrm{CH}_{3} \mathrm{OH}$ lines).

The scientific questions possible to target with (sub)mm interferometry in star formation research cover nearly all physical and chemical processes one may think of. Here, I will present some of the most important applications in the framework of this European network: obviously molecular outflows, then the closely related accretion disks, furthermore the question of fragmentation and early sub-structure formation, as well as astrochemical applications. Last but not least, I will give an outlook of the future (sub)mm array ALMA.

\section{Molecular outflow}

\section{Low-mass outflows}

Shocks associated with molecular outflows and jets have first been detected in the 1950s [33, 31. Later in the 1970s, first non-Gaussian line-wing emission from molecular $\mathrm{CO}$ was detected 43 . While molecular outflows were originally not predicted by theory, after their observational detection it was obvious that they are a crucial ingredient to remove angular momentum during star formation. Today, it is well established that star-forming regions of all masses and luminosities drive molecular outflows, however, it is still debated whether the outflow driving mechanisms are always the same (e.g., 2]). In low-mass star formation the current paradigm includes the formation of a centrifugally supported accretion disk and associated outflows/jets driven by magneto-centrifugal acceleration (e.g., [53]). 
The text-book example of a low-mass outflow is the one observed toward the class 0 source HH211. The region was observed in $\mathrm{CO}(1-0)$ with the PdBI covering both outflow lobes in a large mosaic [29. They found a collimated jet-like component at velocities $>10 \mathrm{~km} / \mathrm{s}$ with respect to the $v_{\mathrm{lsr}}$, and a less collimated, spatially broader component at lower velocities. This morphology was interpreted in the framework of jet-entrainment where a collimated high-velocity jet entrains additional gas from the surrounding envelope. More recent follow-up observations in several transitions of $\mathrm{SiO}$ and higher excited $\mathrm{CO}$ lines revealed the jet-like nature of this outflow in even more detail (Fig. 1. [35, 51]). Corresponding $\mathrm{SiO}$ knots are closer to the driving source in more highly excited lines indicating higher temperatures at the knot shock fronts. Furthermore, $\mathrm{SiO}(8-7) /(5-4)$ line ratios decrease with distance from the driving source which is indicative of a density gradient in the surround gas core. Last but not least, it was found that the $\mathrm{SiO}$ emission exhibits always higher velocities than $\mathrm{CO}$ at the same projected position of the molecular outflow (Fig. 1, 51]). This is interpreted again in the jet-entrainment picture where the $\mathrm{SiO}$ emission is closer to the axis of the primary beam and hence at higher velocities whereas the $\mathrm{CO}$ emission traces entrained gas further outside. However, one has to keep in mind that even such jet-like molecular components are not the primary jet ejected by the protostar-disk system, but that even the observed jet-like molecular gas is already entrained gas. Observed optical jets show far higher velocities of the order a few $100 \mathrm{~km} / \mathrm{s}$ [48. Different jetentrainment processes have been proposed, e.g., entrainment via bow-shocks at the head of the jet, turbulent entrainment via Kelvin-Helmholtz instabilities or wide-angle winds, for more details see a recent discussion of the various entrainment possibilities [2].

Fig. 1. The left panel presents the recent SMA observations of the molecular jet observed at submm wavelengths in the $\mathrm{SiO}(8-7)$ and $\mathrm{CO}(3-2)$ lines [51. Blue and red contours show the line emission as labeled in each panel and the grey-scale outlines the shocked $\mathrm{H}_{2}$ emission. The grey-contours in the middle panel outline $\mathrm{NH}_{3}$ emission, and the $\mathrm{SiO}(8-7)$ spectrum in the bottom panel is averaged over the central $10^{\prime \prime}$ of the jet. The right panel shows the corresponding position-velocity diagram with $\mathrm{SiO}(8-7)$ in grey-scale and $\mathrm{CO}(3-2)$ in contours.

While the jet-entrainment scenario is relatively well established today for low-mass star formation, other interesting topics are under discussion. For example, optical slit spectroscopy observations toward DG Tau found rotation of the optical jet around its outflow axis [3, and complementary mm interferometer investigations revealed the corresponding accretion disk around the protostar with the same rotation orientation as the jet 69 . This is strong support for the tight disk-jet connection and that the jets have to be launched from the protostar-disk interface. The main two competing theories for the jet-launching are magneto-centrifugal disk winds emanated from an extended 
inner disk region (e.g., [53]) and the $\mathrm{X}$-wind theory predicting that the jetlaunching region is close to the inner disk truncation radius, at the so-called X-point 63. Ray et al. (2006) [54 presented new jet-rotation observations that did not actually trace the disk-launching region, but that allowed to extrapolate at what distance from the protostar the disk is most likely to be launched. Although these observations are not finally conclusive, they are consistent with current disk-wind models favoring a more extended disk-region as jet-launching site.

\section{High-mass outflows}

The picture of molecular outflows from high-mass star-forming regions has changed considerably over the last few years mainly based on interferometric observations at $\mathrm{mm}$ wavelengths. Since the mid-90s, increasing evidence arose that molecular outflows are ubiquitous phenomena in high-mass star formation as well (e.g., 61, 11). However, early single-dish mapping studies of high-mass outflows claimed that the collimation degree of massive outflows is lower than known for low-mass outflows (e.g., 60, 56]). This was interpreted as support for alternative formation scenarios going to higher-mass stars (e.g., [18, 66]). In contrast to this claim, single-dish observations with better angular resolution revealed that the observed collimation degrees of massive outflows are consistent with those from their low-mass counterparts as soon as the larger distances and lower spatial resolution of such regions are properly taken into account [11. As the next step, interferometric observations of some high-mass star-forming regions clearly resolved the previously chaoticly appearing single-dish observations into multiple outflow systems from various members of the evolving high-mass protocluster (e.g., [10, 12, 28, 67, 27, 26]). Figure 2 shows the multiple collimated outflows in the massive star-forming region IRAS $05358+3543$ as an example. These observations support a picture for massive star formation where the qualitative physical processes are similar to those in low-mass star formation, i.e., accretion processes mediated by accretion disks and associated outflows/jets. The main differences are 1) the clustered mode of high-mass star formation which produces observational problems and the need for high-spatial resolution, and 2) that the most relevant physical processes are quantitatively much stronger, i.e., higher accretion rates, higher outflow rates and masses, larger luminosities, more UV radiation, etc. On a cautionary note, it should be stressed that the observed molecular outflows rarely exceed regions where the central objects are more massive than $30 \mathrm{M}_{\odot}$. Therefore, going to higher-mass regions, we may encounter more severe changes than so far observed.

\section{Accretion disks}

Angular momentum conservation and rotation of molecular cores always predicted that during star formation gas and dust has to assemble in disk-like 
Fig. 2. The left and middle panel present PdBI observations toward the young massive star-forming region IRAS $05358+3543$ [10. The grey-scale shows the shocked $\mathrm{H}_{2}$ emission and the blue/red contours the $\mathrm{CO}(1-0)$ and $\mathrm{SiO}(2-1)$ emission, respectively. The arrows and large ellipses guide the eye for the various outflow directions, and the pentagons show additional $\mathrm{H}^{13} \mathrm{CO}^{+}(1-0)$ peak positions. The top-left inlay zooms into the marker box with high-velocity $\mathrm{CO}$ emission in contours and $3 \mathrm{~mm}$ continuum in grey-scale. The right panel presents the corresponding $1.2 \mathrm{~mm}$ continuum (grey-scale) and blue/red $\mathrm{CO}(2-1)$ emission (contours) observed previously with the IRAM $30 \mathrm{~m}$ single-dish telescope.

structures around the forming central protostar. However, evidence was lacking for a long time. First observational indications for the existence of accretion disks was based on single-dish multi-wavelengths observations of TTauri stars. For example, analyzing the spectral energy distributions of a sample of low-mass protostars from optical to mm wavelength, the mm dust continuum emission implied such high column densities that in spherical symmetry one would not have detected the sources in the optical or infrared [5. However, since these sources were optical visible TTauri stars, spherical symmetry was excluded, and disk-symmetry appeared the most likely way to solve this problem. Then in the mid-90th, Hubble Space Telescope observations revealed accretion disks in Orion as absorption shadows against the strong background radiation (e.g., 47]).

Since accretion disks are dense, flattened dust and gas condensations, they have on average relatively low temperatures of the order a few $10 \mathrm{~K}$. Therefore, if one wants to learn more about their structure, dynamics and kinematics, the mm and submm bands are the wavelength regimes of choice. Furthermore, with disk sizes of the order $100 \mathrm{AU}$ at typical Taurus distances of $150 \mathrm{pc}$, a spatial resolution of $\leq 1^{\prime \prime}$ is necessary to resolve any substructure. Although disks in massive star formation are likely larger of the order $1000 \mathrm{AU}$, their average distance is $\geq 2 \mathrm{kpc}$, and again one needs sub-arcsecond spatial resolution to study such objects. Hence (sub)mm interferometry is the tool of choice.

Observational studies have focused over recent years on several issues in accretion disk studies, among them are 1) dust evolution, the formation of larger grains and the way to planet formation, 2) kinematic studies of disks and their stability, 3 ) chemical properties and chemical complexity in accretion disks. While chemistry will be discussed in $\$ 5$ important results for topics 1) and 2) will be summarized here.

It is known for centuries that the planets of our solar system are located approximately within the ecliptic plane originating from the initial accretion disk, and that their velocity structure can be well explained by the Keplerian laws. Because the formation of planets is so tightly linked with accretion disks, dust evolution and the formation of larger objects is essential for our understanding of the earth history. One way to study dust properties is to observe 
accretion disks at different wavelength and then investigate their spectral energy distribution. In the Rayleigh-Jeans limit, which is a good approximation at $\mathrm{mm}$ wavelength, the mm continuum flux $S$ scales with $\nu^{2+\beta}$, where $\nu$ is the frequency and $\beta$ the dust opacity index $\left(\tau \propto \nu^{\beta}\right)$. For normal interstellar grains $\beta$ is $\sim 2$ 34. With increasing grain sizes this index $\beta$ decreases continuously. Single-dish studies of a sample of accretion disk candidates found lower values of $\beta$ and claimed already grain growth in these sources [5]. However, with single-dish studies alone, the results are ambiguous because a decreasing spectral index can also be mimicked by an increasing optical depth within the disk. Only spatially resolved interferometric disk studies later could determine the disk density structure, infer their optical depth and hence better determine that grain growth actually takes place in accretion disks [4, 49. Incorporating more sophisticated dust and radiative transfer models, one can infer density distributions, temperature distributions and dust composition in even better detail (e.g., 20, 77, 49, 58,).

An interesting new way to study dust and disk properties is interferometry at mid-infrared wavelength. The Very Large Telescope Interferometer (VLTI) offers the MIDI instrument [44] which allows two-baseline interferometry at wavelengths between 8 and $12 \mu \mathrm{m}$ covering a strong silicate band. In one such study, it was found that the inner disk regions has a higher degree of crystallization than usually observed in the interstellar medium [72].

As the evolutionary next step, the dust around debris disks can be studied at (sub)mm wavelengths as well. For example, Wilner et al. 74] observed the $1.3 \mathrm{~mm}$ dust continuum emission toward the Vega debris disk, and they found two dust condensations between 60 and $100 \mathrm{AU}$ from the exiting star. Such a dust distribution can be explained by the dynamical influence of an unseen planet of a few Jupiter masses in a highly eccentric orbit that traps dust in principal mean motion resonances [74.

To investigate disk kinematic properties, one needs spectral line observations of the disks. The best accessible target for such kind of studies are TTauri stars in the relatively evolved class II stage of protostellar evolution [1]. At this stage, most of the original protostellar envelope has already been dispersed, and the accretion disks remains rather isolated and undisturbed for observational studies (In younger regions, one suffers from confusion between disk and envelope contributions to the observed spectral lines.). A good example of CO emission from a protostellar disk is the study of DM Tau with the PdBI (Fig. 3. [30]). They resolve the velocity structure of the disk finding a velocity distribution along the disk axis consistent with Keplerian rotation (i.e., the centrifugal force equals the gravitational force $F_{\text {cen }}=\frac{m v^{2}}{r}=F_{\mathrm{g}}=\frac{G m_{*} m}{r^{2}} \rightarrow v=\sqrt{\frac{G m_{*}}{r}}$. This also implies that the observed linewidth increases getting closer to the central star (see Fig. 3). In a more statistical sense, larger samples of accretion disk sources were observed at high spatial resolution, and typical Keplerian velocity structure was found in many objects (e.g., 64). 


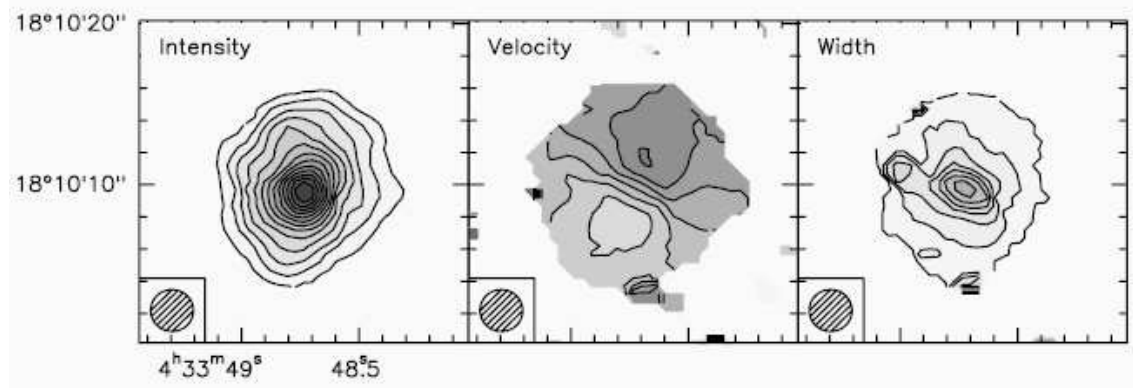

Fig. 3. CO(1-0) emission from the Keplerian protostellar disk DM Tauri 30. The left panel shows the integrated intensity, the middle panel the velocity field and the right panel the line width.

However, also different disk structures have been observed. For example, the disk around the prototypical Herbig Ae star AB Aurigae shows a central depression in the cold dust and gas emission and a non-Keplerian velocity profile $\left(v=r^{-0.4 \pm 0.01}\right)$ [52, 45]. Possible explanations for such deviations from more typical disk structures could be either the formation of a low-mass companion or planet in the inner disk, or a much earlier evolutionary phase where the Keplerian motion is not yet established. Hence, non-Keplerian velocity structures are expected for very young as well as more evolved accretion disks.

While the picture for low-mass accretion disks has tremendously evolved over the last decade, progress in massive disk studies has been significantly slower. This is again partly due to the on average larger distances of massive star-forming regions, and due to the clustered mode of massive star formation that complicates the spatial isolation of massive accretion disks. However, an additional problem arises from the fact that massive star formation proceeds much faster than low-mass star formation (of the order $10^{5} \mathrm{yr}$ compared to a few times $10^{6}$ years), and that evolved massive stars will relatively quickly dissipate the original accretion disks. Therefore, it is unlikely to find a massive accretion disk in a similarly evolved and exposed state like the low-mass TTauri stars, but we have to search for these objects in the much younger and deeply embedded evolutionary stages. This implies, that molecular line as well as dust continuum emission is usually not solely attributable to an accretion disk, but that we always have additional contributions from the larger-scale core and envelope emission. In many cases, this envelope emission can dominate any line and continuum study. One technical advantage of interferometers is that they filter out the emission on large spatial scales because they are not sensitive to structures $\theta$ corresponding to baseline lengths $D$ below the shortest separation of two baselines $\left(\theta \sim \frac{\lambda}{D}\right.$, for more details see, e.g., 68, 70]). Therefore, we can filter out large parts of the envelope emission, nevertheless, not all emission is usually filtered out, and one still 
suffers from confusion between genuine disk and surrounding core emission. To overcome this difficulties, one is interested to observe molecular lines that are usually weak or not found in the envelope, but that should be strong in the inner disk regions. Typical hot core molecules like $\mathrm{CH}_{3} \mathrm{CN}, \mathrm{HCOOCH}_{3}$, $\mathrm{C}^{34} \mathrm{~S}, \mathrm{HN}^{13} \mathrm{C}$ or torsionally excited $\mathrm{CH}_{3} \mathrm{OH}$ transitions are good candidates for such lines. However, observations over the last few years have shown that in many sources exclusively one or the other tracer only allows rotational studies, whereas other candidate spectral lines are either too weak, optically thick or maybe have not been formed in the chemical network yet. For more details on these problems see a summary in a recent review [8]. This difficulties complicate statistical studies of massive accretion disks, and our current knowledge is based on only a few selected examples.

One of the best known massive disk candidates is within the high-mass star-forming region IRAS 20126+4104 [22, 21, 78, 24]. Observations in various spectral lines clearly identify a velocity gradient perpendicular to the molecular outflow/jet, and the velocity structure is consistent with Keplerian rotation 24]. Based on the Keplerian motion, the mass of the central object is estimated to "only" $7 \mathrm{M}_{\odot}$, hence a large fraction of the observed luminosity of the order $10^{4} \mathrm{~L}_{\odot}$ has to be due to accretion luminosity [41]. Based on the existing data, it is suggested that this source is still actively accreting and may well form a much more massive star at the of its evolution [24]. For most other massive star-forming regions of higher luminosity, no clear signatures of Keplerian rotation have been found so far. The current interpretation of larger-scale rotation signatures is that such larger-scale entities with sizes of a few $1000 \mathrm{AU}$ rather resemble rotating, probably infalling toroid that may harbor more genuine accretion disk at their so far unresolved centers 23. The likely most extreme case of rotating and infalling signatures has been observed toward the well-known very luminous $\left(\sim 10^{6} \mathrm{~L}_{\odot}\right)$ ultracompact HII region G10.6. Rotational infalling signatures were observed on larger scales in the molecular gas $\left(\mathrm{NH}_{3}\right.$ lines, 65$)$, and on much smaller scales similar signatures were identified in the ionized gas $\left(\mathrm{H}_{\alpha}\right.$ recombination line, [38]). These observations likely trace a larger-scale in-spiraling structure that continues from the molecular to the ionized gas. In classical Hir region theory that would not have been possible because the formed HII region should expand by the pressure and not allow any further infall and accretion. The observations of G10.6 allow a different scenario, where the gravity still dominates the structure of the UCHII region (not yet the pressure), and hence accretion through the evolving HiI regions may be possible for some time [39, 40].

\section{Fragmentation}

Since its first determination in 1955 [57], the rather universal validity of the Initial Mass Function (IMF) has been confirmed in many ways. For a recent compilation see [25]. One of the essential question in general star formation 


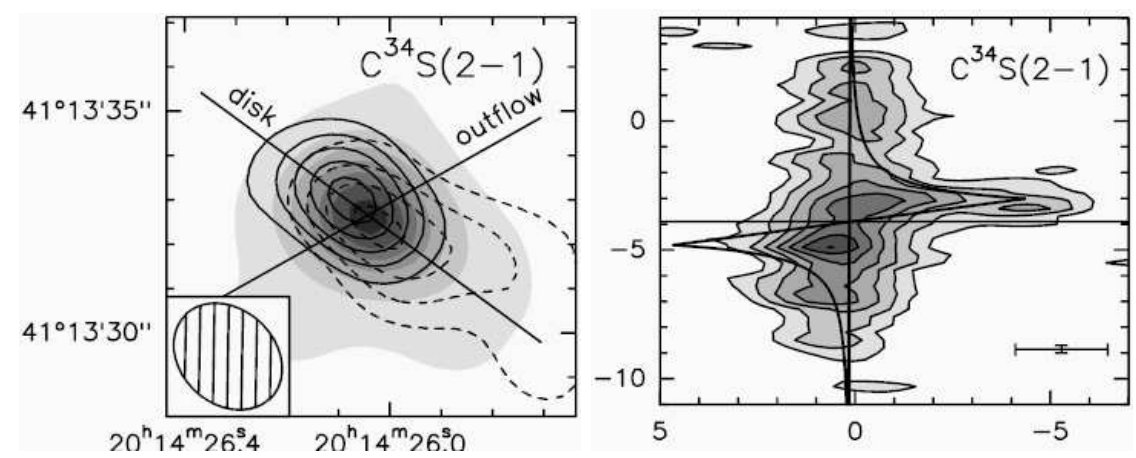

Fig. 4. Keplerian disk emission toward the HMPO IRAS 20126+4104 24]. The left panel shows in grey-scale the $3 \mathrm{~mm}$ continuum emission and in full and dashed contours the blue- and red-shifted $\mathrm{C}^{34} \mathrm{~S}(2-1)$ emission. The outflow and disk axis are indicated. The right panel presents a position velocity diagram (X-axis offset, Y-axis velocity). The full line shows the expected emission of a Keplerian disk.

research is how and at what evolutionary stage the shape of the IMF gets established. Turbulent fragmentation theories predict that the turbulence right at the onset of fragmentation processes already produces core mass functions with the same power law like the IMF, and that by applying star formation efficiencies these core mass functions directly convert into the later observed IMF [50, 46]. Contrary to that, other groups argue that the the early starforming gas clumps fragment down to many cores of more or less the Jeans mass $\left(\sim 0.5 \mathrm{M}_{\odot}\right)$, and that the IMF will be formed from that stage via competitive accretion from previously unbound gas [19, 17.

In the low-mass regime, dust continuum studies with bolometer arrays installed on single-dish telescopes revealed the core mass distributions in nearby regions like $\rho$ Ophiuchus and Orion. The power-law distributions $d N / d M \propto M^{-\alpha}$ of the pre-stellar dust condensations resembles the Salpeter IMF with typical values of $\alpha$ between 2 and 2.5. While this finding is support for the turbulent fragmentation scenario, it only covers a small range of the IMF, and it is necessary to expand such studies to higher-mass regions. Several groups investigated with the same single-dish instruments samples of massive star-forming regions, and the derived cumulative mass distributions of their samples resemble the Salpeter IMF as well [62, 73, 55, 6]. However, since these massive star-forming regions are at about an order of magnitude larger distances, these single-dish studies do not resolve individual protostars but they average over the whole forming protocluster. Hence, these cumulative mass distributions rather resemble protocluster mass functions, and it is far from clear whether they can set any constraints on the formation of the IMF. Therefore, again high spatial resolution is required to resolve individual protostars within the forming protoclusters. So far, only one study resolved and imaged enough sub-sources within a massive star-forming re- 
gions that a derivation of a core mass function appeared meaningful. The two massive gas condensation in IRAS $19410+3543$ were resolved in $1.3 \mathrm{~mm}$ continuum observations with the PdBI into 24 sub-sources (Fig. 5, 9]), and the resulting core mass function again has a power-law distribution with $\alpha \sim 2.5$. Although the statistics are still poor, and a few caveats have to be taken into account in this analysis (Are all dust continuum peaks of protostellar nature? Is the assumption of the same temperature for all sub-sources justified?), it is exciting that all studies so far find core mass distributions resembling the IMF, indicating that turbulent fragmentation processes really are important. However, especially in the high-mass regime, we cannot draw any definitive conclusions yet. Larger source samples observed at high angular resolution are required to base the results on solid grounds. Furthermore, it will be necessary to investigate the temperature structure of the sub-sources in detail, and we need additional spectral line data to determine complementary virial masses and thus establish that the observed sub-sources are really bound and not transient structures.

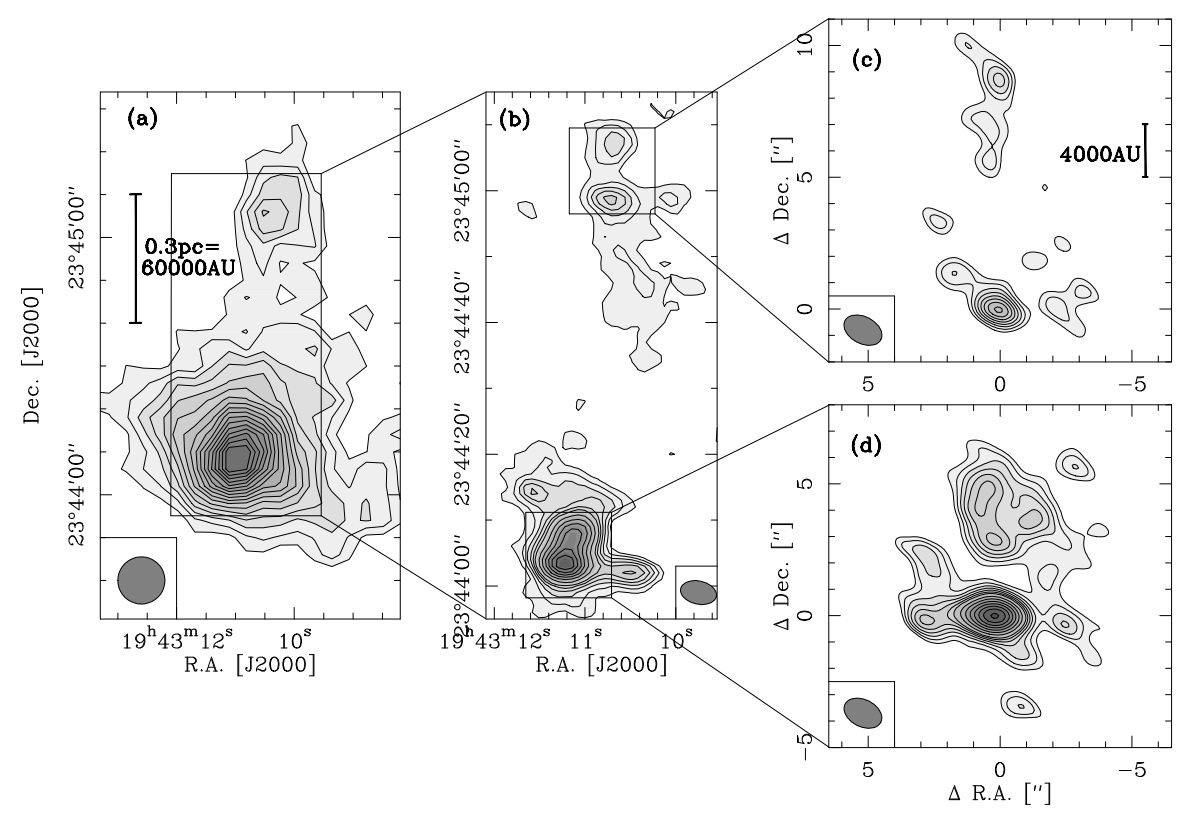

Fig. 5. Single-dish and interferometer mm dust continuum observations toward the young high-mass star-forming region IRAS 19410+2336 [9]. The left panel shows the $1.2 \mathrm{~mm}$ map obtained with the IRAM $30 \mathrm{~m}$ telescope. The middle and right panel present 3 and $1.3 \mathrm{~mm}$ continuum images from the PdBI with increasing spatial resolution. The synthesized beams are shown at the bottom-left of each panel. The resolution difference between the left and right panels are an order of magnitude. 

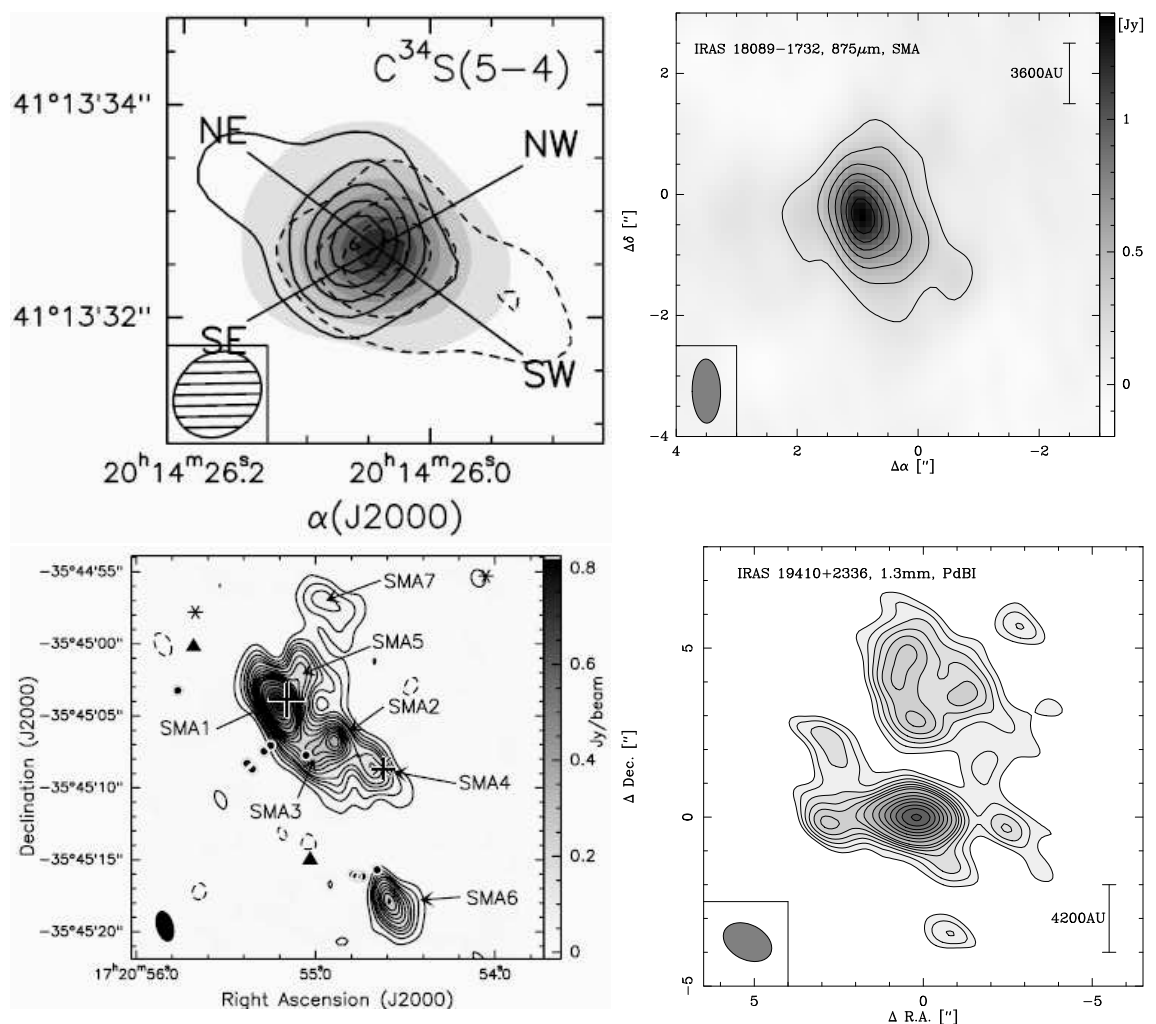

Fig. 6. Interferometric high-spatial-resolution (sub)mm dust continuum images of sources at comparable evolutionary stage and luminosity but with very different fragmentation characteristics. The top-left panel shows IRAS 20126+4104 (grey: $1.3 \mathrm{~mm}$ continuum, contours $\mathrm{C}^{34} \mathrm{~S}(5-2), 1.7 \mathrm{kpc}, 24$ ), the top-right is IRAS 18089$1732(875 \mu \mathrm{m}, 3.6 \mathrm{kpc}, 15)$, the bottom-left is $\operatorname{NGC6334I(N)}(1.3 \mathrm{~mm}, 1.7 \mathrm{kpc}, 37$, the bottom-left is IRAS $19410+2336(1.3 \mathrm{~mm}, 2.1 \mathrm{kpc}, 9])$. The synthesized beams are shown at the bottom-left of each image.

Another observational curiosity of several high-mass star-forming regions, that appear very similar from previous single-dish observations (They are at similar distances, should be at approximately the same evolutionary stage, and have comparable luminosities and other massive star-forming tracers like masers and outflows.), is that they reveal very different sub-structures when observed at high angular resolution. Figure 6 shows a few interferometric example studies of regions where one would have expected comparable fragmentation results. However, some of the regions show only a single massive central source, even at the highest angular resolution (Fig. 6 top row), whereas other sources exhibit many sub-sources as expected from a star-forming cluster (Fig. 6 bottom row). While the latter is less of a surprise since we know that massive stars almost always form in clusters, finding only a single peak 
in other regions is more difficult to explain. While all these studies are sensitivity limited and usually are not capable to trace any condensation below approximately $1 \mathrm{M}_{\odot}$, one would nevertheless expect to find at least some intermediate-mass objects in the vicinity of the central object. One important additional fact is that toward most regions deeply embedded near-infrared clusters have been found, and hence they are no isolated objects. The existence of an embedded near-infrared protocluster in the vicinity of a massive forming star may be interpreted in the direction that the low-mass stars form first and the high-mass objects later (see also [4]). However, currently we do not understand why various apparently similar massive star-forming regions show such a diverse fragmentation behavior. Is it only an observational bias and the selected sources may be in fact not as similar as we believe, or may there be different paths massive star-forming regions can fragment in the first place?

\section{Chemistry}

Astrochemistry is a steadily rising topic over recent years. Large-scale chemical mapping surveys of molecular clouds (e.g., 7, 71 and line surveys toward high-mass hot molecular cores [59, 32, both conducted with single-dish instruments, have already been conducted for more than two decades. However, studying the chemical small-scale diversity of hot molecular cores has been a difficult task because the spectral bandpasses available for most existing interferometers were mostly too small to cover enough molecular lines from various species in a reasonable amount of time (a noteworthy exception is [16]). This has changed significantly since the advent of the SMA with its two times $2 \mathrm{GHz}$ bandwidth in the upper and lower sideband [36, and similar capabilities to be available very soon at the PdBI and CARMA. As a prime showcase of the spatial chemical complexity of hot molecular cores, here a recent investigation of Orion-KL will be presented.

The Orion-KL region at a distance of $450 \mathrm{pc}$ is the closest and prototypical hot molecular core. With the advent of the SMA, it obviously became one of the early targets of this instrument, and the region was mapped at arcsec spatial resolution in the $865 \mu \mathrm{m}$ submm wavelength band [14]. The $4 \mathrm{GHz}$ bandpass covered more than 150 spectral lines from at least 13 species, 6 isotopologues and 5 vibrational excited states within a single observation run. From a chemical point of view, the observations included nitrogen- as well as oxygen-bearing species, shock-tracer like $\mathrm{SiO}$ and $\mathrm{SO}$, more than $40 \mathrm{CH}_{3} \mathrm{OH}$ lines, and many complex molecules like $\mathrm{CH}_{3} \mathrm{CH}_{2} \mathrm{CN}$ or $\mathrm{HCOOCH}_{3}$. While this chemical diversity is interesting in itself, it also allows to study various physical properties in detail, e.g., the $\mathrm{SiO}$ data can be used for a kinematic analysis of the molecular outflow(s) in the region, and the large number of $\mathrm{CH}_{3} \mathrm{OH}$ lines (ground state as well as torsionally excited $v_{t}=1,2$ lines) are an ideal tool to investigate the temperature structure of the whole region. Furthermore, the 
data also allowed to derived the first sub-arcsecond resolution dust continuum map of this region [13, clearly differentiating the central power house source $I$ from the hot molecular core, detecting source $n$ for the first time in cold dust emission, and identifying a new protostellar source SMA1, which may be the driver of one of the outflows in this complex star-forming region.

Fig. 7. SMA submm spectral line observations toward Orion-KL 14. The figure shows representative images from various molecular species labeled in each panel. Full contours show positive emission, dashed contours negative features due to missing short spacings. The stars mark the locations of source $I$, the hot core peak position, the newly identified source SMA1 and source $n$ (see bottom-right panel).

Figure 7 shows a small number of line images from the whole dataset to outline the chemical complexity of the Orion-KL hot molecular core. While $\mathrm{SiO}$ traces the collimated outflow structure centered on source $I$, all other species show a more complex morphology. Nitrogen-bearing species like $\mathrm{CH}_{3} \mathrm{CN}$ or $\mathrm{CH}_{3} \mathrm{CH}_{2} \mathrm{CN}$ exhibit the kind of horse-show morphology which was know for the hot core already from early $\mathrm{NH}_{3}$ observations (e.g., [75]). In contrast, the oxygen-bearing molecules, foremost $\mathrm{CH}_{3} \mathrm{OH}$, are relatively speaking weaker there, but they show a strong additional peak toward the south-west, the so-called compact ridge. This southern region is believed to be the interface of one of the molecular outflows (as traced in $\mathrm{SiO}$ ) with the ambient cloud, and hence the $\mathrm{CH}_{3} \mathrm{OH}$ is strongly excited in this shocked interface region. Other molecules, e.g. $\mathrm{C}^{34} \mathrm{~S}$, show a mixture of these morphologies and sometimes even an additional peak positions toward the north-west associated with the infrared-source IRC6. The main lesson one may take home from this spatial molecular differences is that massive star-forming regions are far more complex than one generally assumes, and that one always has to take into account various physical and chemical processes like heating, outflows, shocks or chemical evolution if one wants to model such regions accurately. While single-dish surveys give a good average molecular inventory of such regions, they do not allow to set more detailed constraints. Interferometric imaging surveys are needed to investigate their structures in depth.

\section{Outlook and Future}

Interferometry at mm wavelength has been conducted for more than one and a half decades by now, but only recently with the advent of the SMA, the submm spectral window started to be accessible for high-spatial-resolution observation. Both wavelength regimes can be considered as close siblings where a range of scientific questions can be targeted by both, but where also some other questions are unique for each band. For example, hot molecular cores are by definition strong in the submm regime whereas colder and younger 
regions are more easily studied in the mm band. Therefore, none of the bands is obsolete, but they complement each other well. The main facilities in the northern hemisphere are and will be the SMA, the PdBI and CARMA, and they will be the sites of many exciting science over the coming decade.

Nevertheless, the upcoming Atacama Large Millimeter Array (ALMA), which will be built within the next few years in Chile by a worldwide collaboration of North-America, Europe and Japan, will supersede the already existing instruments in many ways by orders of magnitude. At an altitude of $\sim 5000 \mathrm{~m}$, ALMA will consist of fifty $12 \mathrm{~m}$ dishes (25 provided by Europe and 25 by the USA), combined with a few $7 \mathrm{~m}$ antennas provided by the Japanese partners to supplement the short spacing information (the Atacama Compact Array, ACA). The baselines range will be from $15 \mathrm{~m}$ and $15 \mathrm{~km}$, and the receivers are expected to cover all earth-accessible spectral windows between 30 and $950 \mathrm{GHz}$. The spectral first-light bands are expected to be around 100, 230, 345 and $690 \mathrm{GHz}$, and the bandpass will be broad with $8 \mathrm{GHz}$ and dual polarization capability. Combining these specs, the anticipated $350 \mathrm{GHz}$ continuum sensitivity is $1.4 \mathrm{mJy}$ in 1 second integration time. For more detailed descriptions of the specs and the scientific capabilities of ALMA, one may visit http://www.eso.org/projects/alma/.

The requirements on the ALMA capabilities have been driven by mainly three scientific topics:

- Detect CO or CII in a normal galaxy at $\mathrm{z}=3$ in less than 24 hours $(\rightarrow$ sensitivity).

- Image protostars and proto-planetary disks around sun-like stars at 150pc (Taurus) to study kinematics, chemistry, magnetic fields and tidal gaps created by forming planets ( $\rightarrow$ spatial resolution down to 10mas).

- Good imaging quality down to $0.1^{\prime \prime}$ resolution $(\rightarrow$ spatial resolution and uv-coverage/number of antennas).

In the framework of the topics discussed in this chapter, a few likely highlights should be mentioned. With the highest spatial resolution, we will be able to probe the central launching regions of the outflow and jets and hence observationally much better constrain the physical processes governing these energetic processes of energy and momentum transfer. As outlined above, disk/planet research has been one of the main drivers for ALMA, and we will witness the planet-formation processes within the young accretion disks (e.g., [76]). For massive accretion disk studies, the advent of ALMA will be important in many ways: the high spatial resolution will help to better disentangle the clustered sub-structure of the regions, the even broader bandpasses will allow to sample many spectral lines simultaneously and thus give a broad range of analysis tools within single observations, and the high sensitivity will give access to the weaker, optically thin and less abundant lines, which are likely the best candidate lines to differentiate the genuine disk emission from the surrounding envelopes. Regarding future fragmentation studies, ALMA will allow to study larger source-samples in a consistent manner, which is needed to draw statistically significant constraints. Furthermore, the instan- 
taneous extremely good uv-coverage provides an order of magnitude better image fidelity, which is very important to derive credible core-mass distributions in young massive star-forming regions. Last but not least, spectral imaging surveys will gain significant more momentum: Again based on the broad bandpasses and high sensitivity, such surveys can be done is a far more consistent manner for large source samples covering various evolutionary stages and extending from the high-mass to the low-mass regime. This will help to differentiate much better the chemical evolution depending on mass as well as on time. Furthermore, combining the good uv-coverage of the main ALMA array with the short spacing information from the ACA, we will not have the problem of negative features caused by the missing short spacings as shown in Fig. 7 These missing flux problem so far severely hampered correct column density determinations. Overcoming these difficulties is crucial for accurate abundance determinations which are one of the important parameters in any evolutionary chemical network. In addition, it is expected that spectral imaging surveys will greatly help to identify new molecules and thus potentially pave the way to find the first bio-molecules in space.

Although this chapter could only roughly outline the potential of (sub) $\mathrm{mm}$ interferometry, it has to be stressed that this field is extremely vibrant and, many exciting questions are waiting to be investigated. The progress over the last two decades has been enormous, and we currently have a number of excellent facilities to study many important astrophysical/astrochemical questions. In addition to this, the future is bright with ALMA on the horizon. This new facility will add another quantum leap to (sub)mm interferometry and probably nearly all fields of astrophysical research.

H.B. acknowledges financial support by the Emmy-Noether-Program of the Deutsche Forschungsgemeinschaft (DFG, grant BE2578).

\section{References}

1. Andre, P., Ward-Thompson, D., Barsony, M.: From Prestellar Cores to Protostars: the Initial Conditions of Star Formation. Protostars and Planets IV p. 59 (2000)

2. Arce, H., Shepherd, D., Gueth, F., Lee, C.F., Bachiller, R., Rosen, A., Beuther, H.: Molecular Outflows from Low- to High-Mass Star Formation. In: PPV, astro-ph/0603071 (2006)

3. Bacciotti, F., Ray, T.P., Mundt, R., Eislöffel, J., Solf, J.: Hubble Space Telescope/STIS Spectroscopy of the Optical Outflow from DG Tauri: Indications for Rotation in the Initial Jet Channel. ApJ576, 222-231 (2002)

4. Beckwith, S.V.W., Henning, T., Nakagawa, Y.: Dust Properties and Assembly of Large Particles in Protoplanetary Disks. Protostars and Planets IV p. 533 (2000)

5. Beckwith, S.V.W., Sargent, A.I., Chini, R.S., Guesten, R.: A survey for circumstellar disks around young stellar objects. AJ99, 924-945 (1990) 
6. Beltrán, M.T., Brand, J., Cesaroni, R., Fontani, F., Pezzuto, S., Testi, L., Molinari, S.: Search for massive protostar candidates in the southern hemisphere. II. Dust continuum emission. A\&A447, 221-233 (2006)

7. Bergin, E.A., Langer, W.D.: Chemical Evolution in Preprotostellar and Protostellar Cores. ApJ486, 316 (1997)

8. Beuther, H.: Physics and chemistry of hot molecular cores. In: IAU proceedings 237, astro-ph/0610411 (2006)

9. Beuther, H., Schilke, P.: Fragmentation in massive star formation. Science 303, 1167-1169 (2004)

10. Beuther, H., Schilke, P., Gueth, F., McCaughrean, M., Andersen, M., Sridharan, T.K., Menten, K.M.: IRAS 05358+3543: Multiple outflows at the earliest stages of massive star formation. A\&A387, 931-943 (2002)

11. Beuther, H., Schilke, P., Sridharan, T.K., Menten, K.M., Walmsley, C.M., Wyrowski, F.: Massive molecular outflows. A\&A383, 892-904 (2002)

12. Beuther, H., Schilke, P., Stanke, T.: Multiple outflows in IRAS 19410+2336. A\&A408, 601-610 (2003)

13. Beuther, H., Zhang, Q., Greenhill, L.J., Reid, M.J., Wilner, D., Keto, E., Marrone, D., Ho, P.T.P., Moran, J.M., Rao, R., Shinnaga, H., Liu, S.Y.: Subarcsecond Submillimeter Continuum Observations of Orion KL. ApJ616, L31-L34 (2004)

14. Beuther, H., Zhang, Q., Greenhill, L.J., Reid, M.J., Wilner, D., Keto, E., Shinnaga, H., Ho, P.T.P., Moran, J.M., Liu, S.Y., Chang, C.M.: Line Imaging of Orion KL at $865 \mu \mathrm{m}$ with the Submillimeter Array. ApJ632, 355-370 (2005)

15. Beuther, H., Zhang, Q., Sridharan, T.K., Chen, Y.: Testing the Massive Disk Scenario for IRAS 18089-1732. ApJ628, 800-810 (2005)

16. Blake, G.A., Mundy, L.G., Carlstrom, J.E., Padin, S., Scott, S.L., Scoville, N.Z., Woody, D.P.: A lambda = 1.3 millimeter Aperture Synthesis Molecular Line Survey of Orion Kleinmann-Low. ApJ472, L49 (1996)

17. Bonnell, I., Larson, R., Zinncker, H.: The Origin of the Initial Mass Function. In: PPV, astro-ph/0603447 (2006)

18. Bonnell, I.A., Bate, M.R., Zinnecker, H.: On the formation of massive stars. MNRAS298, 93-102 (1998)

19. Bonnell, I.A., Vine, S.G., Bate, M.R.: Massive star formation: nurture, not nature. MNRAS349, 735-741 (2004)

20. Calvet, N., D’Alessio, P., Hartmann, L., Wilner, D., Walsh, A., Sitko, M.: Evidence for a Developing Gap in a 10 Myr Old Protoplanetary Disk. ApJ568, 1008-1016 (2002)

21. Cesaroni, R., Felli, M., Jenness, T., Neri, R., Olmi, L., Robberto, M., Testi, L., Walmsley, C.M.: Unveiling the disk-jet system in the massive (proto)star IRAS 20126+4104. A\&A345, 949-964 (1999)

22. Cesaroni, R., Felli, M., Testi, L., Walmsley, C.M., Olmi, L.: The disk-outflow system around the high-mass (proto)star IRAS 20126+4104. A\&A325, 725-744 (1997)

23. Cesaroni, R., Galli, D., Lodato, G., Walmsley, C., Zhang, Q.: Disks around Young O-B (Proto)Stars: Observations and Theory. In: PPV, astro-ph/0603093 (2006)

24. Cesaroni, R., Neri, R., Olmi, L., Testi, L., Walmsley, C.M., Hofner, P.: A study of the Keplerian accretion disk and precessing outflow in the massive protostar IRAS 20126+4104. A\&A434, 1039-1054 (2005) 
25. Corbelli, B., Palla, F., Zinnecker, H. (eds.): The Initial Mass Function 50 years later (2005)

26. Davis, C.J., Varricatt, W.P., Todd, S.P., Ramsay Howat, S.K.: Collimated molecular jets from high-mass young stars: IRAS 18151-1208. A\&A425, 981$995(2004)$

27. Garay, G., Brooks, K.J., Mardones, D., Norris, R.P.: A Triple Radio Continuum Source Associated with IRAS 16547-4247: A Collimated Stellar Wind Emanating from a Massive Protostar. ApJ587, 739-747 (2003)

28. Gibb, A.G., Hoare, M.G., Little, L.T., Wright, M.C.H.: A detailed study of G35.2-0.7N: collimated outflows in a cluster of high-mass young stellar objects. MNRAS339, 1011-1024 (2003)

29. Gueth, F., Guilloteau, S.: The jet-driven molecular outflow of HH 211. A\&A343, 571-584 (1999)

30. Guilloteau, S., Dutrey, A.: Physical parameters of the Keplerian protoplanetary disk of DM Tauri. A\&A339, 467-476 (1998)

31. Haro, G.: Herbig's Nebulous Objects Near NGC 1999. ApJ115, 572-+ (1952)

32. Hatchell, J., Thompson, M.A., Millar, T.J., MacDonald, G.H.: A survey of molecular line emission towards ultracompact HII regions. A\&AS133, 29-49 (1998)

33. Herbig, G.H.: The Spectra of Two Nebulous Objects Near NGC 1999. ApJ113, $697-+(1951)$

34. Hildebrand, R.H.: The Determination of Cloud Masses and Dust Characteristics from Submillimetre Thermal Emission. QJRAS24, 267 (1983)

35. Hirano, N., Liu, S.Y., Shang, H., Ho, P.T.P., Huang, H.C., Kuan, Y.J., McCaughrean, M.J., Zhang, Q.: SiO J = 5-4 in the HH 211 Protostellar Jet Imaged with the Submillimeter Array. ApJ636, L141-L144 (2006)

36. Ho, P.T.P., Moran, J.M., Lo, K.Y.: The Submillimeter Array. ApJ616, L1-L6 (2004)

37. Hunter, T.R., Brogan, C.L., Megeath, S.T., Menten, K.M., Beuther, H., Thorwirth, S.: Millimeter Multiplicity in NGC 6334 I and I(N). ApJ649, 888-893 (2006)

38. Keto, E.: An Ionized Accretion Flow in the Ultracompact H II Region G10.6-0.4. ApJ568, 754-760 (2002)

39. Keto, E.: On the Evolution of Ultracompact H II Regions. ApJ580, 980-986 (2002)

40. Keto, E.: The Formation of Massive Stars by Accretion through Trapped Hypercompact H II Regions. ApJ599, 1196-1206 (2003)

41. Krumholz, M., Klein, R., McKee, C.: Radiation-hydrodynamic simulations of collapse and frgamentation in massive protostellar cores. ArXiv Astrophysics e-prints: astro-ph/0609798 (2006)

42. Kumar, M.S.N., Keto, E., Clerkin, E.: The youngest stellar clusters. Clusters associated with massive protostellar candidates. A\&A449, 1033-1041 (2006)

43. Kwan, J., Scoville, N.: The nature of the broad molecular line emission at the Kleinmann-Low nebula. ApJ210, L39-L43 (1976)

44. Leinert, C.: Scientific observations with MIDI on the VLTI: present and future. In: W.A. Traub (ed.) New Frontiers in Stellar Interferometry, Proceedings of SPIE Volume 5491. Edited by Wesley A. Traub. Bellingham, WA: The International Society for Optical Engineering, 2004., p.19, pp. 19-+ (2004) 
45. Lin, S.Y., Ohashi, N., Lim, J., Ho, P.T.P., Fukagawa, M., Tamura, M.: Possible Molecular Spiral Arms in the Protoplanetary Disk of AB Aurigae. ApJ645, 1297-1304 (2006)

46. Mac Low, M., Klessen, R.S.: Control of star formation by supersonic turbulence. Reviews of Modern Physics 76, 125-194 (2004)

47. McCaughrean, M.J., O'dell, C.R.: Direct Imaging of Circumstellar Disks in the Orion Nebula. AJ111, 1977-+ (1996)

48. Mundt, R., Buehrke, T., Solf, J., Ray, T.P., Raga, A.C.: Optical jets and outflows in the HL Tauri region. A\&A232, 37-61 (1990)

49. Natta, A., Testi, L.: Grain Growth in Circumstellar Disks. In: D. Johnstone, F.C. Adams, D.N.C. Lin, D.A. Neufeeld, E.C. Ostriker (eds.) ASP Conf. Ser. 323: Star Formation in the Interstellar Medium: In Honor of David Hollenbach, pp. $279-+(2004)$

50. Padoan, P., Nordlund, A.: The Stellar Initial Mass Function from Turbulent Fragmentation. ApJ576, 870-879 (2002)

51. Palau, A., Ho, P.T.P., Zhang, Q., Estalella, R., Hirano, N., Shang, H., Lee, C.F., Bourke, T.L., Beuther, H., Kuan, Y.J.: Submillimeter Emission from the Hot Molecular Jet HH 211. ApJ636, L137-L140 (2006)

52. Piétu, V., Guilloteau, S., Dutrey, A.: Sub-arcsec imaging of the AB Aur molecular disk and envelope at millimeter wavelengths: a non Keplerian disk. A\&A443, 945-954 (2005)

53. Pudritz, R., Ouyed, R., Fendt, C., Brandenburg, A.: Disk winds, jets and outflows: Theoretical and computational foundations. In: PPV, astro-ph/0603592 (2006)

54. Ray, T., Dougados, R., Bacciotti, F., Eisloeffel, J., Chrysostomou, A.: Toward Resolving the Outflow Engine: An Observational Perspective. In: PPV, astroph/0605597 (2006)

55. Reid, M.A., Wilson, C.D.: High-Mass Star Formation. I. The Mass Distribution of Submillimeter Clumps in NGC 7538. ApJ625, 891-905 (2005)

56. Richer, J.S., Shepherd, D.S., Cabrit, S., Bachiller, R., Churchwell, E.: Molecular Outflows from Young Stellar Objects. Protostars and Planets IV p. 867 (2000)

57. Salpeter, E.E.: The Luminosity Function and Stellar Evolution. ApJ121, 161 (1955)

58. Schegerer, A., Wolf, S., Voshchinnikov, N.V., Przygodda, F., Kessler-Silacci, J.E.: Analysis of the dust evolution in the circumstellar disks of T Tauri stars. A\&A456, 535-548 (2006)

59. Schilke, P., Groesbeck, T.D., Blake, G.A., Phillips, T.G.: A Line Survey of Orion KL from 325 to $360 \mathrm{GHz}$. ApJS108, 301 (1997)

60. Shepherd, D.S., Churchwell, E.: Bipolar Molecular Outflows in Massive Star Formation Regions. ApJ472, 225 (1996)

61. Shepherd, D.S., Churchwell, E.: High-Velocity Molecular Gas from High-Mass Star Formation Regions. ApJ457, 267 (1996)

62. Shirley, Y.L., Evans, N.J., Young, K.E., Knez, C., Jaffe, D.T.: A CS J=5-4 Mapping Survey Toward High-Mass Star-forming Cores Associated with Water Masers. ApJS149, 375-403 (2003)

63. Shu, F.H., Najita, J.R., Shang, H., Li, Z.Y.: X-Winds Theory and Observations. Protostars and Planets IV p. 789 (2000)

64. Simon, M., Dutrey, A., Guilloteau, S.: Dynamical Masses of T Tauri Stars and Calibration of Pre-Main-Sequence Evolution. ApJ545, 1034-1043 (2000) 
65. Sollins, P.K., Zhang, Q., Keto, E., Ho, P.T.P.: Spherical Infall in G10.6-0.4: Accretion through an Ultracompact H II Region. ApJ624, L49-L52 (2005)

66. Stahler, S.W., Palla, F., Ho, P.T.P.: The Formation of Massive Stars. Protostars and Planets IV p. 327 (2000)

67. Su, Y., Zhang, Q., Lim, J.: Bipolar Molecular Outflows from High-Mass Protostars. ApJ604, 258-271 (2004)

68. Taylor, G.B., Carilli, C.L., Perley, R.A. (eds.): Synthesis Imaging in Radio Astronomy II (1999)

69. Testi, L., Bacciotti, F., Sargent, A.I., Ray, T.P., Eislöffel, J.: The kinematic relationship between disk and jet in the DG Tauri system. A\&A394, L31-L34 (2002)

70. Thompson, A.R., Moran, J.M., Swenson Jr., G.W.: Interferometry and Synthesis in Radio Astronomy, 2nd Edition. Interferometry and synthesis in radio astronomy, Jr. 2nd ed. New York : Wiley (2001)

71. Ungerechts, H., Bergin, E.A., Goldsmith, P.F., Irvine, W.M., Schloerb, F.P., Snell, R.L.: Chemical and Physical Gradients along the OMC-1 Ridge. ApJ482, $245-+(1997)$

72. van Boekel, R., Min, M., Leinert, C., Waters, L.B.F.M., Richichi, A., Chesneau, O., Dominik, C., Jaffe, W., Dutrey, A., Graser, U., Henning, T., de Jong, J., Köhler, R., de Koter, A., Lopez, B., Malbet, F., Morel, S., Paresce, F., Perrin, G., Preibisch, T., Przygodda, F., Schöller, M., Wittkowski, M.: The building blocks of planets within the 'terrestrial' region of protoplanetary disks. Nature432, 479-482 (2004)

73. Williams, S.J., Fuller, G.A., Sridharan, T.K.: The circumstellar environments of high-mass protostellar objects. I. Submillimetre continuum emission. A\&A417, 115-133 (2004)

74. Wilner, D.J., Holman, M.J., Kuchner, M.J., Ho, P.T.P.: Structure in the Dusty Debris around Vega. ApJ569, L115-L119 (2002)

75. Wilson, T.L., Gaume, R.A., Gensheimer, P., Johnston, K.J.: Kinematics, Kinetic Temperatures, and Column Densities of $\mathrm{NH}_{3}$ in the Orion Hot Core. ApJ538, 665-674 (2000)

76. Wolf, S., D'Angelo, G.: On the Observability of Giant Protoplanets in Circumstellar Disks. ApJ619, 1114-1122 (2005)

77. Wolf, S., Padgett, D.L., Stapelfeldt, K.R.: The Circumstellar Disk of the Butterfly Star in Taurus. ApJ588, 373-386 (2003)

78. Zhang, Q., Hunter, T.R., Sridharan, T.K.: A Rotating Disk around a High-Mass Young Star. ApJ505, L151-L154 (1998) 
This figure "05358_elba.jpg" is available in "jpg" format from: http://arxiv.org/ps/astro-ph/0611706v1 
This figure "orion.jpg" is available in "jpg" format from: http://arxiv.org/ps/astro-ph/0611706v1 
This figure "palau_f1.jpg" is available in "jpg" format from: http://arxiv.org/ps/astro-ph/0611706v1 
This figure "palau_f3.jpg" is available in "jpg" format from: http://arxiv.org/ps/astro-ph/0611706v1 